\title{
Brownfield identification: different approaches for analysing data detected by means of remote sensing
}

\author{
V. Ferrara \\ Department of Electronic Engineering, \\ University of Rome "La Sapienza”, Italy
}

\begin{abstract}
This paper aims to show different techniques for identifying brownfields and for monitoring based on remote sensing. If brownfields are associated with urban wastes such as the demolition of buildings, dangerous materials or contaminants will be involved with them. Hazard and health risks increase, so the administration policy needs to detect, at an early stage, dangerous materials or contaminants and to manage the cleaning and recovery of these land areas. A detailed classification of the land and the identification of these typologies of brownfields can be carried out by means of hyperspectral remote sensing using the airborne MIVIS (Multispectral Infrared and Visible Imaging Spectrometer) sensor of the Laboratory of CNR (LARA). Thanks to MIVIS, we can detect many different materials. The subsequent integration of data into GIS allows identification of brownfields through our specific software tools that determine the contours of objects and evaluate the statistical parameters of the processed images. Moreover, other GIS tools correlate data in order to evaluate the extent and derivative parameters of the same brownfields. When brownfields are associated with urban or sub-urban abandoned industrial properties or a land with neglected vegetation, i.e. with the evident characteristic of land abandoned or under-used; then the previous method is useless. These brownfields are missing specific hazardous substances or contaminants and buildings and are generally in good condition. For these last cases, an alternative process should be implemented. A higher geometric resolution than MIVIS should be used, putting in evidence small objects in the soil and so IKONOS or QuickBird satellite imagery are required. In this case, brownfield identification will be carried out by means of object-oriented algorithms and the mutual relationship between them.
\end{abstract}

Keywords: monitoring, multispectral sensors, MIVIS, IKONOS, QuickBird, GIS. 


\section{Introduction}

The identification and location of brownfield areas are essential processes to help authorities implement their environmental policies. The recovery of those urban and suburban zones actually abandoned or underused decreases health risks when brownfields include hazardous substances. The process of mapping the roofing material and the detection of hazardous substances represent the first step to assessing the risk level, which is related to the study area under examination. Furthermore, when disused structures such as parking lots, commercial or industrial buildings, industrial hangars, etc., are detected, their recovery can turn the planning of administrative resolutions into economical advantages. In effect, brownfields of urbanized sites, where the network of utility structures are already in place, can be restored to productive use both from an environmental and economic viewpoint, thanks to the lower soil use. On one hand, the investigation must be concerned with the detection of hazardous material, and on the other in the individuation of disused buildings (or land areas now abandoned). In order to detect each specific target, one should acquire different images. This depends on economical convenience and on technical aspects: the required spatial resolution, the elaboration techniques of data, data correlation and specific software algorithms.

In our previous work, Atturo et al. [1], we showed a method for hazardous substances detection. That is realized from the Laboratory LARA (Airborne Laboratory for Environmental Research) of the Italian National Research Council (CNR) by means of the airborne MIVIS (Multispectral Infrared and Visible Imaging Spectrometer) sensor. Results of this activity are combined with software post-elaborations delimiting brownfield areas of crumbling buildings and urban wastes. GIS (Geographic Information System) tools then correlate the relevant data in order to evaluate extension and derivative parameters of the same brownfields [10]. These parameters are important for the elaboration of indicators that can be used within the framework of sustainable planning and development.

A procedure that identifies different types of areas, such as abandoned car parks, uses satellite imagery to a high spatial resolution, such as Quickbird, IKONOS and SPOT. Typically, the procedure compares data collected by consecutive images that produce significant indicators, such as the status indicator of neglect. Obviously, both preventive location and boundaries individuation of the area under examination should be carried out. The tools of many GIS can resolve the last problem, often by the semi-automatic mode.

All processes based on remote sensing (included airborne sensors) have advantages when both periodic investigation of large areas and automatic or semi-automatic analysis are implemented. The cost of these images is comparatively high, but the multiuse of the applications in different scientific fields of environmental management can reduce their economic burden. Administrative authorities investigate different environmental aspects such as traffic, bus line networks, air pollution, planning, etc. On the other hand the evaluation of brownfield recovery needs the knowledge of correlated data. 
Frequently the easiest means of detection of these is by remote sensing. So, a relevant image can be used for the evaluation of several parameters and indicators. Multispectral images with different resolutions must be elaborated before using them in classification and correlation techniques.

GIS adding further data for each specific location, identified by a pixel of the image, allowing multidimensional processes. If GIS is open to external tools, like Terrapack ${ }^{\circledR}[3-6]$, algorithms can be experimented to find new solutions for better identification of substances or structures in automatic/unsupervised mode.

The results of this work show progress in our procedure for finding sites. At first, we will present the procedure based on the classification of hazardous substances and brownfield detection. Later on, we will examine another process whose design lines are: structure detection, definition of category of everyday use, evaluation of usage continuity, brownfield identification according to its use degree.

\section{Hyperspectral classification}

Thanks to the collaboration of researchers of the Italian National Research Council (CNR), in our previous work [1] we presented the hyperspectral classification technique. We refer to that article for fuller details. This paper reviews only the essential parts.

MIVIS is an airborne platform system included in the LARA Project (Airborne Laboratory for Environmental Research) of CNR. It consists of a modular scanning system that operates with a high spectral and geometric resolution (for instance, precision at soil $4 \mathrm{~m} \mathrm{x} 4 \mathrm{~m}$ from $2000 \mathrm{~m}$ of altitude). It uses four spectrometers and 102 spectral bands within the $0.43 \mu \mathrm{m}$ and $12.7 \mu \mathrm{m}$ wavelengths (from visible to thermal infrared spectrum). This large number of spectral bands allows analysis of both chemical and physical parameters of the Earth's surface. In effect the process bases itself on the spectral signature concept. So, relevant classification characterizes many ground features such as vegetation, bare soils, building structures, road paving, wastes and so on.

The retro-diffused energy coming from the atmosphere towards the sensor can be important; consequently these effects of atmosphere are corrected by using IRC (Internal Radiometric Calibration) [13].

Owing to the detection methods of a remote sensing system, which varies several observation parameters (frequency, polarization and incidence angle of the scenes), many and different effects must be decreased. Specifically, alignment problems, contrasts equalization and compensation of the effects of high frequency attenuation. Radiometric correction and data conversion are realized at ground level by means of the MIDAS (Multispectral Interactive Data Processing and Analysis System) station.

A supervised classification technique called Spectral Angle Mapper (SAM) allows us to optimize the similarities (in terms of "angle") existing between image spectra and reference spectra [7]. The latter can be defined either in the laboratory or in the field or extracted interactively from the imagery. 
In order to classify the whole area, some training areas or reference spectra resulting from specific "Regions Of Interest" (ROIs) or spectral databank are fixed as inputs of the SAM algorithm.

Integration of the hyperspectral data in GIS is the last step of the process. It allows the superimposition of morphological, chemical and physical features on the geographical maps. This integration is fundamental for the data interpretation by means of interactive reading.

\section{Brownfield: wear state of structures}

When the brownfield consists of buildings in a state of wear, it is likely to require the identification of hazardous substances. They stand out owing to collapsed roofs and walls. Obviously the presence of hazardous substances isn't a peculiar characteristic of a collapse: in a lot of towns and cities there are buildings still inhabited with asbestos-cement for example. So, a different indicator should be evaluated, one linked to the geometrical aspect of area.

The presence and expansion of this brownfield can be risky involving environmental contamination; consequently the investigation on their location and identification should assume a higher priority in the administration policy.

Mapping of the roofing material and the detection of hazardous substances are the first steps to evaluate the risk level of the area under examination. Remote sensing classification techniques, such as hyperspectral classification, allow the identification of risk of large areas.

In order to provide a more accurate risk level assessment, interpretation of remotely sensed data must always be associated to in situ measurements. A calibration is often decisive in distinguishing among different kinds of situation.

After territorial classification by means of hyperspectral remote sensing detection, recognition of brownfield should ensue from a series of consecutive and derivative steps: discrimination between brownfield and habitable structures, extension evaluation, possible risk assessment, nearness evaluations and so on.

Imagery of airborne remote sensing is correlated to others providing a more detailed recognition and a lower uncertainty of the geometric features. These other images are normally originated from a different source and frequently have a different resolution, they are geo-referred as remote sensing images and so overlaid over the same area.

\subsection{Brownfield identification}

Collapsed buildings and waste lands show irregular contours of the structure together with discontinuities of the surface coverings. A remote sensing in the Visible spectrum allows us to evaluate these irregular geometrical features. Nowadays, three-dimensional maps [2,11] are available. They are the best inputs for software tools that show buildings pulled down. However, their use increases the cost. The process described in this paper makes use of two-dimensional maps only. They are more common and are already included in the midst of hyperspectal data. Sometimes a better resolution could be used and new georeferred images acquired, but at a lower cost. 


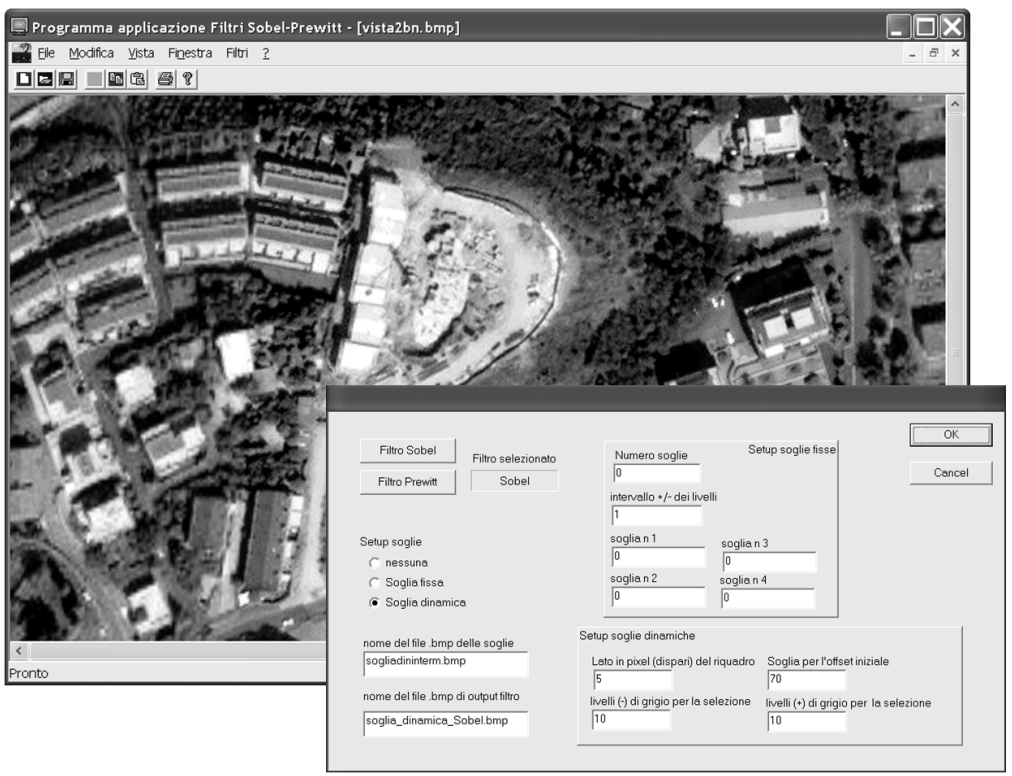

a)

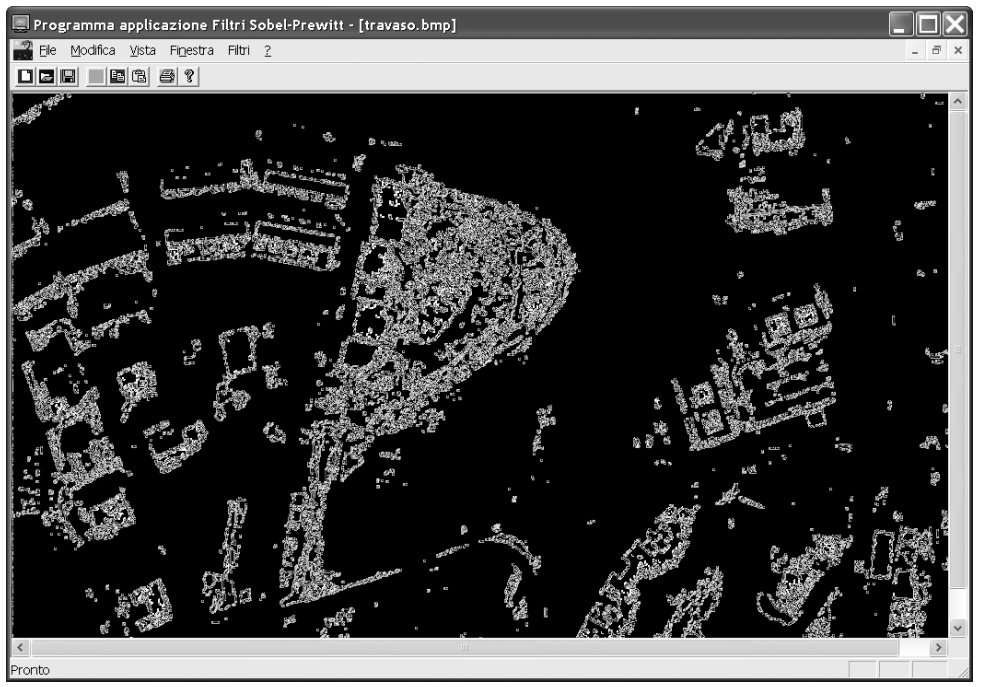

b)

Figure 1: Extraction of contours by means of Prewitt's filter application. The figure shows input window (a) and the results of the first elaboration step (b) by using dynamical thresholds. 
Obviously the evaluation method of irregular geometry will have been optimized in terms of timing, tools and reliability degree of response.

The software procedure uses two different investigations whose results can be combined to increase the degree of reliability of the identification. The first one emphasizes the building contours, whereas the second one evaluates the surface homogeneity. These are surely two indicators that can be used to distinguish between good conservation and the wear state of a building.

Contours are analyzed by means of classical filtering techniques that use kernels of Sobel or Previtt. Each kernel is a $3 \times 3$ matrix that must be used for convolution operation together with the matrix of the image $[12,16]$.

Hyperspectral classification identifies areas where there are hazardous substances covering the soil or roof. In this mode contour analysis can be applied to delimited zones to obtain the reduction of elaboration cost. Convolution operates on a map in the individuated area and in the Visible spectrum [17].

Images (normally a grey-level map) of a higher resolution than data from MIVIS need to be geo-referred and superimposed on the map. In order to improve edge identification, the same images are filtered by means of an algorithm based on intensity threshold. In particular we get the histogram of intensity levels. The statistic of number and position of the intensity peaks defines the dynamical thresholds that we apply the filter with. In the case of the wear state of a building, the peaks of the histogram are not evident and spread with a high value of standard deviation. The subsequent convolution between kernel and images (filtered) outlines the boundaries.

The irregular sequence of the contour, as discontinuities and interruptions, is an indicator of the wear state of the building. Unfortunately different materials, covering the soil and roofs, could show the same grey-level intensity. If they are adjoining, anomalous discontinuities together with wrong lines appear as a result of the convolution. In order to reduce false responses, we intersect data of hyperspectral classification with a grey-level map. So, we separate pixels of the same grey-level but classified in a different way. Next, we generate the vectors of the contours. GIS tools can use both vector and raster data and identify brownfield by means of the evaluation of discontinuities, rate of variation in the area under examination (index of surface homogeneity) and standard deviation.

\section{Brownfield: disused structures}

Abandoned industrial properties and land with neglected vegetation are examples of lands abandoned or under-used, which are not identifiable by the procedure described previously. They are missing specific hazardous substances or contaminants and buildings are generally in a good state.

For these cases, an alternative process should be implemented. Literature proposed a methodological approach that satisfies the essential prerequisites allowing classification of complex objects and to use high quality data $[9,15]$. Following the essential lines of that method we implemented our process through which to extend the spectrum of identified cases. 
Even if MIVIS shows high spatial resolution, e.g. four meters in the Visible spectrum, the specific brownfield sites identification and their location could require higher resolution data. The IKONOS satellite collects both multispectral and panchromatic images simultaneously. The geometrical resolution for soil is four meters and one meter respectively. If the acquisition is shot from nadir, the resolution improves at eighty centimetres for the panchromatic and thirty-two decimetres for the multispectral. In order to further improve to one meter the resolution of multispectral data, data fusion should be applied.

As well as IKONOS, QuickBird uses eleven bits for imagery enhancing radiometric resolution. QuickBird is a high resolution satellite operated by DigitalGlobe. It collects image data to sixty-one centimetres of resolution (from nadir, whereas it collects seventy-two centimetres from $25^{\circ}$ off-nadir). The images are panchromatic with bands from $0.45 \mu \mathrm{m}$ to $0.9 \mu \mathrm{m}$ of wavelength: blue, green, red and near IR. The revisit time is one day for IKONOS and from one day to 3.5 days, depending on latitude, in the case of the QuickBird satellite. Unfortunately imagery of these satellites show highly textured data. This is due to a source of noise whose increase generates a decrease in accuracy of land use classification, based on a per-pixel method. However, urban brownfield sites must be defined suitably by the different elements of which they consist buildings, roads and so on. In other words the interpretation of an image is not per-pixel, but by means of identification of the objects and by their reciprocal connections.

Classification is definable per-field; it needs a preventive delimitation of imagery characterized by homogenous patches of land. So, image segmentation sets up a phase of the procedure because it allows the division of suitable imagery into homogenous areas. In the case of multiresolution, the segmentation is realized in each band with the specific weight [14].

The process consists therefore of three phases: the multiresolution segmentation, the assignation of each segment to a class (realizing a supervised classification) and finally individuation of brownfield class. Obviously the optimal situation is to reduce to two the number of classes. MVIS data can help supervised classification adding more radiometric resolution bands.

In order to define typologies of brownfield classes, segments, such as industrial buildings and the state of parking and roads, are combined using fuzzy logic and the analysis of the hierarchical connection $[8,18]$.

However, in this procedure, the characteristic "disuse" of a structure, such as an industrial building, is not deducible with certainty. It could be determined, for instance, by examining frequency of use of a parking lot. If it is empty, without a car, on a working day, it is likely it has fallen into disuse. Confirming the absence of cars in two or more weekdays by means of multitemporal imagery the reliability of brownfield detection improves.

\section{Discussion}

Remote sensing has been used to implement two different methods; each of which has its own advantages. Specifically, the object-oriented approach includes a higher level of interpretation activity, but it is complicated from 
numerous factors such as organization of test sites, timing acquisition and so on. For instance, time acquisition is important for the interpretation of an empty parking area, but it furthermore characterizes the aspect of the entire image in terms of the presence and extension of shadows. These are a noise source, particularly important in the correspondence of narrow streets.

Obviously, multitemporal acquisition, stereo-imagery (IKONOS supplies it), orthophotos or different thematic maps that are superimposed on the imagery under study can help to discriminate objects and to decrease erroneous interpretations (failures of the method).

When brownfield sites are identified, both the additional source data and the GIS tools are involved in a new step of "interpretation" with the aim of evaluating indicators of the brownfield state: all useful information for redevelopment and reuse of area. Each additional image is correlated to others, geo-referred as the first one, overlaid to the same geographical area, even if having a different resolution.

\section{Conclusions and acknowledgments}

More remote sensing techniques have been used, identifying different brownfield sites. Some brownfields are characterized by the presence in the area of hazardous materials, some are only a structure or land that is under-used or abandoned. In the first case, during monitoring of brownfield sites, MIVIS hyperspectral data together with GIS showed good results.

The use of MIVIS data allows identification of numerous and different materials by means of classification techniques. In order to locate brownfields, our algorithms intersect the data of classified imagery with data in the Visible spectrum. The procedure analyzes specifically the information associated to a single pixel. A different approach to investigation has been implemented to detect urban structures not-used or under-used. The last procedure, based on an object-oriented technique, solves the classification by means of both fuzzy logic and analysis of the hierarchical connection. The two methods are complementary. The second process is totally "supervised", and it denies itself the characteristic "automatic" of the first one. By assembling together the methods, the number of identifications has increased, adding a lot of different typologies of brownfields. Thus, classification of potential urban brownfields in the study zone produced promising results.

Further test areas will be examined in our next study on confirming reliability of each procedure and to verify more interaction between the two methods.

The author wishes to thank Dr Lorenza Fiumi and his team for support and contribution on MIVIS data. Moreover he thanks Professor Cesare M. Ottavi who coordinated the development of the GIS Terrapack ${ }^{\circledR}$.

\section{References}

[1] Atturo, C., Cianfrone, C., Ferrara, V., Fiumi, L., Fontinovo, G. \& Ottavi, C.M., Remote Sensing Detection Techniques for Brownfield Identification 
and Monitoring by GIS Tools, Proceedings of 3rd international Conference Brownfields III, ed. Wit Press Southampton, Boston, pp. 241-250, 2006

[2] Richman, A., Hamilton, A., Arayici, Y., Counsell, J. \& Tkhelidze, B., Remote Sensing, LIDAR, automated data capture and the VEPS project, Proceedings of the Ninth International Conference on Information Visualisation (IV'05), IEEE, 2005

[3] Ferrara, V., Gariazzo, C., Ottavi, C.M. \& Poli, U., An Integrated approach to the pollution hazard analysis and management, Proceedings of RISK ANALYSIS II, ed. Brebbia, WIT Press, Southampton, pp. 23-32, 2000

[4] Ferrara, V. \& Ottavi, C.M., Structures and organisation of an information tool dedicated to simulation and management of environmental risks, Proceedings of RISK ANALYSIS III, ed. Brebbia, WIT Press, Southampton, pp. 213-222, 2002

[5] Ferrara, V., Integrated data and utilities to support sustainable planning, Proceedings of Sustainable Planning \& Development, Wit Press Southampton, Boston, pp. 375-382, 2003

[6] Ferrara, V. \& Guerriero, M., Territorial Information System interoperability: a design improving interaction in an emergency, Proceedings of Risk Analysis IV, Wit Press Southampton, Boston, pp. 475484, 2004

[7] Boardman, J. W. \& Kruse, F. A., Automated spectral analysis: a geological example using AVIRIS data, North Grapevine Mountains, Nevada, Proc. of the Tenth Thematic Conference on Geologic Remote Sensing, San Antonio Texas USA, I, pp. 407-418, 1994.

[8] Shackelford, A. K., 2003. A Hierarchical fuzzy classification approach for high-resolution multi-spectral data over urban areas. IEEE Trans. Geosci. Rem. Sens, 41(9), pp. 1920-1932, 2003.

[9] Barnsley, M. \& Barr, S. A graph-based structural pattern recognition system to infer land use from fine spatial resolution land cover data. Computers, Environment and Urban Systems, 21(1997), pp. 209-225.

[10] Thomas, M.R., A GIS-based decision support system for brownfield redevelopment, Landscape and Urban Planning, pp. 58:7-23, 2002.

[11] Gatti, M., Stereoscopia ottica ASTER ed interferometria differenziale SAR a confronto, GEOmedia, GEO4all, 1, pp. 6-13, 2005.

[12] Gonzales, R. \& Woods, R., Digital Image Processing, Addison Wesley Publishing Company, 1992.

[13] Ben-Dor, E., Kruse, F. A., Lefkoff, A. B. \& Banin, A., Comparison of three calibration techniques for utilization of GER 63 channel aircraft scanner data of Makhtesh Raamon Negev, Israel, $P E \& R S$, 60(11), pp.1339-1354, 1994.

[14] Johnsson, K., Segment-based land use classification from SPOT satellite data. Photogrammetric Engineering \& Remote Sensing, Vol. 60, 1, pp.4753, 1994

[15] Banzhaf, E., Hannemann, K., Martini, M., Grescho, V., Netzband, M., Monitoring the urban development with integrated system from RS 
observation and GIS information, Proceedings of Urban Remote Sensing Joint Event, IEEE, 2007

[16] Haykin, S., Adaptive Filter Theory, Prentice-Hall, Inc., 1996.

[17] Parker, J. R., Algorithms for Image Processing and Computer Vision, John Wiley \& Sons, Inc., New York, pp. 23-29, 1997.

[18] Klir, G. J. \& Yuan, B: Fuzzy Sets and Fuzzy Logic. Theory and Applications. Prentice Hall, UpperSaddle River, N J, 1995. 